\title{
NEGATIVE MAGNETORESISTANCE IN THE NEAREST-NEIGHBOUR HOPPING CONDUCTION IN GRANULAR GOLD FILM
}

\author{
B. I. Belevtsev*, E. Yu. Beliayev, Yu. F. Komnik, E. Yu. Kopeichenko \\ B. Verkin Institute for Low Temperature Physics \& Engineering \\ Nat. Acad. of Sci. of Ukraine, Lenin Prosp. 47, 310164, Kharkov, Ukraine
}

\begin{abstract}
The low temperature $(0.5-55 \mathrm{~K})$ conduction of semicontinuous gold film vacuum deposited at $T \approx 50 \mathrm{~K}$ is studied. The film is near the percolation threshold (thickness $3.25 \mathrm{~nm}$ ). Its resistance is extremely sensitive to the applied voltage $U$. At low enough $U$ the film behaves as an insulator (two-dimensional granular metal). In this state the dependences $R(T) \propto \exp (1 / T)$ (for $T \leq 20 \mathrm{~K}$ ) and $R(U) \propto \exp (1 / U)$ ) (for $T \leq 1 \mathrm{~K}$ and $U>0.1 \mathrm{~V}$ ) are observed. Magnetoresistance (MR) is negative and can be described by $\Delta R(H) / R(0) \propto-H^{2} / T$. This negative MR which manifests itself for nearest-neighbour hopping is rather uncommon and, up to now, has not been clarified. The possible mechanisms of such case of negative MR are discussed.
\end{abstract}

\section{INTRODUCTION}

For the last 20 years the significant progress has been achieved in understanding of transport properties of electrons in granular metals (GM) [1-4]. In general case GM represents a random mixture of metal and insulator, i.e. percolating system. In this connection the conduction of GMs is determined in an essential degree by the ratio of the metal volume fraction $p$ and the critical fraction $p_{c}$ (percolation threshold). Three regimes of electron conduction are distinguished [1, 4]: (1) Metallic regime $\left(p>p_{c}\right)$. (2) Dielectric regime $\left(p<p_{c}\right)$. (3) Transition regime $\left(p \simeq p_{c}\right)$. In the dielectric regime the conduction of GMs is by a hopping mechanism in which the charge carriers are transported from one metal grain to another via thermallyactivated tunnelling. It is assumed [4, that in GMs both the nearest-neighbour hopping (NNH) and the variable-range hopping (VRH) [0, 6] are possible.

Because of the activated tunnelling of electrons between grains, the GMs are not classical percolation systems. Sufficiently strong external influence on the probability of activated tunnelling can result in essential change of percolating and conducting properties of GMs. Such

${ }^{*}$ Corresponding author 
influence can be exerted by an applied electrical field $F$. For example, in [7, 8] the pronounced variations of conducting properties of In and Bi granular films under the influence of changing in $F$ was found. The results of these investigations can be considered as the evidence of strong effect of an electrical field on the degree of electron localization in grains. It gives the reason to expect under certain conditions the occurrence of metal-insulator transition (MIT) in GMs under the change of $F$. In the present work we report on detection of such kind of transition in discontinuous gold film near the percolation threshold. It may be assumed that in dielectric regime the external magnetic field must also exert noticeable influence on the probability of activated tunneling between grains. In this connection it should be point out the very interesting results of work [9] in which the negative magnetoresistance (MR) in the NNH conduction for the bulk GM was found. Such case of negative MR for insulators is unusual, and its nature has not been clarified till now.

Ultra thin discontinuous gold films near the percolation threshold were used as the objects of research. The films with sheet resistance $R_{\square}$ about $10 \mathrm{k} \Omega$ were prepared by vacuum deposition onto a cold substrate $(\simeq 50 \mathrm{~K})$. The conduction of films with the above-mentioned values of $R_{\square}$ has appeared to be rather sensitive to the applied voltage $U$. At low enough temperatures, decreasing the magnitude of $U$, we had the opportunity to transfer films from metallic to dielectric regime of conduction. Such transition, besides the essential change of temperature dependences of resistance, is accompanied by the change in the sign of the MR (from positive to negative). The observed transition from the dielectric to the metallic regime of conduction with increase in $U$ is caused by the influence of electrical field on the probability of electron tunnelling between metal grains (percolation clusters). Same as in work [9] we found that negative MR of the investigated film in the dielectric regime has manifested itself in the NNH conduction. We found however the significant distinction from work [9] which will be discussed below. The results obtained can be used for the development of theoretical models of this interesting phenomenon.

The main aim of our paper is to represent and discuss the peculiarities of observed negative $\mathrm{MR}$ in NNH conduction. But for better understanding of properties of investigated system we will shortly describe also its conduction behavior in dielectric regime and main peculiarities of transition from dielectric to metallic state in high enough electrical field.

\section{EXPERIMENTAL PROCEDURES}

The preparation of percolation gold films and the measurement in situ their conducting properties were carried out in a high-vacuum cryostat with ${ }^{3} \mathrm{He}$ and superconducting solenoid. In the following the properties of one of the investigated films are described.f This film with effective thickness $L \approx 3.25 \mathrm{~nm}$ (determined with use of the quartz-crystal monitor) was deposited at the pressure $\approx 10^{-6}$ Pa onto a substrate of a single-crystal sapphire with deposition

${ }^{1}$ For two-dimensional systems $p$ is the metallic surface coverage.

${ }^{2}$ The similar behavior of conductivity was characteristic of other investigated by us gold films, prepared under conditions described in the present paper. 
rate $0.015 \mathrm{~nm} / \mathrm{s}$ at substrate temperature $\simeq 54 \mathrm{~K}$. The purity of initial material was $99.99 \%$. Immediately after preparation the film had resistance $R_{\square} \simeq 4.87 \mathrm{k} \Omega$ at $U \simeq 10 \mathrm{~V}$. For stabilization of the film structural state it was heated to $T \simeq 63 \mathrm{~K}$, then its resistance has decreased to $R_{\square} \simeq 4.5 \mathrm{k} \Omega$. The film sizes were: $2 \times 0.1 \mathrm{~mm}$. The conduction of the film was studied by means of recording of voltage-current characteristics (VCC) at various temperatures (in the range $0.5-55 \mathrm{~K}$ ). In the process the source of a stabilized voltage was used which allowed to change the voltage $U$ in the range from 11 to $0.001 \mathrm{~V}$. In addition to the VCC records, the measurements of $\mathrm{MR}$ in a perpendicular magnetic field $H$ with magnitude up to $\approx 4.5 \mathrm{~T}$ were made.

\section{RESULTS AND DISCUSSION}

\subsection{Conduction in dielectric regime}

The conductivity of the film was essentially non-Ohmic. The temperature variations in $R$ at low $U$ and voltage variations in $R$ at low $T$ were exponentially great. At the same time at large values of $U$ and $T$ the variations in resistance are quite small. At small $U(\leq 0.05 \mathrm{~V})$ the temperature dependence $R(T)$ corresponds to simple exponential dependence

$$
R(T) \propto \exp \left(\frac{E_{0}}{k T}\right)
$$

where $E_{0} \simeq 1.7 \mathrm{meV}$ (curve 1 in Fig. 1). As the applied voltage $U$ increases the functional dependence (1) remains true in some temperature range, but resistance variations are no more exponentially high (curves 2 and 3 in Fig. 1). The simple dependence (1) (Ahrrenius law) signifies that for investigated film the $\mathrm{NNH}$ conduction in dielectric regime takes place. At sufficiently low temperatures $(T<1 \mathrm{~K})$ identical simple exponential dependence $R(U)$ takes place:

$$
R(U) \propto \exp \left(\frac{U_{0}}{U}\right)
$$

where $U_{0} \simeq 2 \mathrm{~V}$ (curve 1 in Fig. 2 ).

As the voltage $U$ increases the temperature dependence $R(T)$ becomes weaker and at large enough voltage $U \approx 10 \mathrm{~V}$ in the range $5-55 \mathrm{~K}$ it corresponds approximately to the relationship $\Delta R(T) \propto \ln T$. The logarithmic dependence of this kind is consistent with the weak localization (WL) and electron-electron-interaction (EEI) effects in two-dimensional (2D) systems [10, 11].

The results obtained testify, thus, that the transition from the dielectric to the metallic regime of conduction occurs in the investigated film as the applied voltage increases. As this takes place the resistance $R_{\square}$ decreases from $\simeq 10^{7} \Omega$ to $\simeq 5 \cdot 10^{3} \Omega$. Along with the change of the temperature dependences of resistance this transition is accompanied by the change of the nature and the sign of the MR (Fig. 3). For low-Ohmic states $\left(R_{\square} \leq 12 \mathrm{k} \Omega\right)$ at rather

high voltages $(\geq 5 \mathrm{~V})$ the MR was positive. It is well known [12, 13, 14, 15], that gold films 
in the regime of WL have positive MR, caused by the strong spin-orbit scattering which is characteristic of gold. We have confirmed that it is also true for low-Ohmic states of the film investigated. To do this we have compared the experimental MR curves with the appropriate theoretical expression 10, 11] and found a good agreement. At doing so we took into account the percolating nature of our film by considering the magnitude of sheet resistance $R_{\square}$ as a fit parameter (s. 13, 16]).

Thus the MR behavior of the investigated film at rather high voltage $(>5 \mathrm{~V})$ is consistent with the WL effects in percolating $2 \mathrm{D}$ systems. As the voltage is decreased the dependence $R(T)$ became stronger (Fig. 1) and approached the exponential dependence (1), i.e. the transition in the dielectric regime of conduction occurred. This transition is accompanied by the change in the sign of MR from positive to negative (Fig. 3).

The observed $R(T)$ and $R(U)$ dependences [the Eqs. (1) and (2)] are in agreement with the current concept of GM conduction in the dielectric regime. It is experimentally established, that the temperature dependences of conductivity $\sigma$ in this regime have the following form: $\sigma \propto \exp \left(-1 / T^{\alpha}\right)$, where $\alpha$ takes different values within the limits 0.25-1.0 [4]. All these dependences have theoretical explanations [1-4] which are based on the assumption that the hopping conduction is determined by the joint contribution of two processes: tunnelling and thermal activation. The case $\alpha=0.25$, for example, is attributed to VRH. The simple exponential dependence $R(T) \propto \exp (1 / T)(\alpha=1)$ observed in our study corresponds to the NNH.

The observed voltage dependence (2) (Fig. 2) also corresponds well to the theoretical dependence $\sigma(U)$ for GMs in the dielectric regime at sufficiently strong electrical fields $F$ and low temperatures [1, 17]:

$$
\sigma \propto \exp \left(-\frac{\chi \phi_{i}}{e F}\right)
$$

where $\chi=\hbar^{-1}\left(2 m \phi_{i}\right)^{1 / 2}$ ( $m$ is the electron mass, $\phi_{i}$ is the effective barrier height), $\chi^{-1}$ is decay length of electron wave function in dielectric. The Eq. (3) is correct only in the moderate fields (eFs $\ll \phi_{i}$, where $s$ is the tunneling distance). In this case the electrical field affects the form and reduces the effective height and width of a potential barrier between grains, but electrons remain localized in grains. In sufficiently large fields electron is emitted as a free particle in dielectric layer between grains (direct field ionization) [17]. In the strong-field limit electrons can become nearly free (the probability of tunnelling is close to unity). In our opinion it is this effect of electrical field on the tunnelling probability that is responsible for the observed transition from the dielectric to the metallic regime of conduction in the gold film as the applied voltage is increased.

It follows from our data that not only the increase in applied voltage but also the increase in temperature can result in transition in temperature dependences of resistance from dielectric to metallic regime of conduction. The general (and, we hope, obvious) picture of influence of $T$ and $U$ is presented in Figure 4. It can be seen that in such representation the equiresistance lines are nearly symmetrized with respect to logarithmic scales of $T$ and $U$. The horizontal 
shading marks the region of positive MR (in remaining part the negative MR takes place). The vertical shading marks the region where the relation (1) is true. Therefore the negative MR occurs in the region where the Arrhenius law (1) holds (and also in adjoining not shaded region, which corresponds to the transition or intermediate regime between the dielectric and metallic regimes).

It can be seen from Fig. 4 that low-Ohmic $\left(<10^{4} \Omega\right)$ states of investigated film can be attained by ways of the increase of temperature (up to $T \geq 20 \mathrm{~K}$ ) or the increase of applied voltage $(U \geq 2 \mathrm{~V})$. In the first case the transition occurs from activated to non-activated tunnelling when the thermal energy $k T$ becomes comparable or larger than the activation energy $E_{0}$ [see Eq. (1)]. The mechanism of applied voltage influence on the resistance was discussed above.

\subsection{Magnetoresistance in the nearest-neighbor hopping conduction}

We have noted above that the MR is negative in dielectric regime. At rather low $U$ the $\mathrm{MR}$ is described by the following square-law dependence: $\Delta R(H) / R=-A(T) H^{2}$ (Fig. 5), where $A(T) \propto 1 / T^{n}, n \approx 1$ (Fig. 6). Therefore the influence of a magnetic field on resistance can be given by

$$
\frac{\Delta R_{\square}(H, T)}{R_{\square}(0, T)}=-B \frac{H^{2}}{T},
$$

The negative MR in the dielectric regime of conduction was revealed in our investigation in the NNH conduction. This is testified by the temperature dependence of conductivity of the investigated film, which is described by the Arrhenius law (Fig. 1), and the exponential voltage dependence $R(U) \propto(1 / U)$ at low enough temperatures (Fig. 2).

Previously the negative MR in the NNH conduction had been observed in [9] in the threedimensional GM (composite systems from $\mathrm{Al}$ and $\mathrm{Al}_{2} \mathrm{O}_{3}$ powders). It was found in this work, that $\Delta R(H) \propto-H$ (i.e. the linear dependence on $H$ ), but the temperature dependence of $\mathrm{MR}$ was not determined. For the investigated percolation gold film we have found the square-law dependence on $H$ and have determined the temperature dependence (see Eq. (4)). ? $^{3}$

For an explanation of negative MR in hopping conduction the interference models [18, 19, 20, 21] are widely used. This approach is based on the considering of the interference of multiply scattered tunneling paths in hopping probability with taking into account the scattering events on the intermediate impurities. The consideration of papers [18, 19, 20], however, is restricted to the VRH, so that their results are not directly applicable to our experimental data. The prediction of model [21] $\left(\Delta R(H) \propto-H^{2} / T\right.$ in conditions of fulfillment of the Arrhenius law $R(T) \propto \exp (1 / T))$ formally is in complete accordance with our results. This model is developed,

${ }^{3}$ We can not properly compare our results with that of [9] since the system studied in that work has (in contrast to our film) very small grain size (diameter is about $3.5 \mathrm{~nm}$ ) and the negative MR was investigated only at high temperatures (at $T=77 \mathrm{~K}$ and $293 \mathrm{~K}$ ). 
however, for lightly doped semiconductors, and it remains unclear, how far it is applicable for the GMs.

We can point out also other two relevant theoretical models [22, 23] of negative $\mathrm{MR}$ in $\mathrm{NNH}$ which could be taken into account at explanation of our results. In the framework of Hubbard model the following mechanism of negative $\mathrm{MR}$ in $\mathrm{NNH}$ conduction is possible [22]. When magnetic field is applied, the electron spins tend to be parallel to the direction of the field owing to the Zeeman effect and hence be parallel to each other. The electron correlation effect is less for the electrons having parallel spins. As a result, the localization length increases with magnetic field. This theory (as was pointed out in [22]) can be applied to GM with small grains, in which the electron energy level separation is large (about $100 \mathrm{~K}$ ). Such level separation can take place in grains with size of a few nanometers. For this reason we beleive that model [22] can not be applied to our film which is near the percolation threshhold and has rather large grains. 4

May be more suitable, in our opinion, is the model [23] of the negative MR in GM with large metal grains and near the percolation threshold (i.e. for the case of small distances between energy levels in grains). The occurrence of negative MR in this model is supposed to be due to the influence of the magnetic field on electron orbital motion, with the result that the change of electron wave functions on the Fermi level occurs. It can lead to the increase of the overlap of wave functions in neighbor grains and, therefore, to the increase of hopping probability. The initial prerequisites for model [23] (large grains and the proximity to the percolation threshold) correspond to the system investigated in our work. The work [23] is, however, only a "prelude". In it only the case $T=0$ is considered and the form of $R(H)$ dependences is not determined. The nature of the negative MR in GM in NNH conduction remains, thus, actually unknown. We hope, that the experimental results obtained in the present work will promote the development of theoretical models of this interesting phenomenon.

\section{CONCLUSION}

The transition from metallic to dielectric regime of conduction has been found for discontinuous gold film at decreasing the electrical field, applied to the sample. The transition is accompanied by the change of the sign of the MR from positive to negative. The negative MR of the film investigated in the dielectric regime manifests itself for the electron hops between the nearest neighbours. The nature of this effect remains unknown. The results of the work can be used for the development of theoretical models of this interesting phenomenon.

\section{Acknowledgments}

The authors are grateful to O. Bleibaum (Technische Universität Magdeburg, Germany), P. Sheng and X.R. Wang (The Hong Kong University of Science and Technology) for fruitful

\footnotetext{
${ }^{4}$ From the MR study of WL effect in metallic regime we have evaluated that the phase coherence length $L_{\varphi} \simeq 20 \mathrm{~nm}$ at $T \simeq 5 \mathrm{~K}$. The metallic grain size must be larger than $L_{\varphi}$ (this is the necessary condition for observation of WL effect).
} 
comments about some aspects of the problem of negative MR in granular dielectric systems in the regime of nearest-neighbour hopping. In addition we thank X. R. Wang for sending us his paper prior to publication. 


\section{References}

[1] B. Abeles, P. Sheng, M. D. Coutts, and Y. Arie, Adv. in Phys. 24 (1975) 407.

[2] P. Sheng and J. Klafter, Phys. Rev. B27 (1983) 2583.

[3] C. J. Adkins, J. Phys.: Cond. Matter 1 (1989) 1253.

[4] P. Sheng, Phil. Mag. B65 (1992) 357.

[5] N. F. Mott and E. A. Davis, Electron processes in non-crystalline materials (Clarendon Press, Oxford ,1979).

[6] B. I. Shklovskii and A. L. Efros, Electronic Properties of Doped Semiconductors (Springer-Verlag, Berlin, 1984).

[7] B. I. Belevtsev, Yu. F. Komnik, A. V. Fomin, Fiz. Niz. Temp. 12 (1986) 821 [Sov. J. Low Temp. Phys. 12 (1986) 465].

[8] B. I. Belevtsev, Yu. F. Komnik, A. V. Fomin, Fiz. Tverd. Tela (Leningrad) 30 (1988) 2773.

[9] A. B. Pakhomov, D. S. McLachlan, I. I. Oblakova, and A M. Virnik, J. Phys.: Cond. Matter 5 (1993) 5313.

[10] P. A. Lee and T. V. Ramakrishnan, Rev. Mod. Phys. 57 (1985) 287.

[11] B. L. Altshuler and A. G. Aronov, in Electron-Electron Interactions in Disordered Systems, eds. A. L. Efros and M. Pollak (North-Holland, Amsterdam, 1985).

[12] G. Dumpich and A. Carl, Phys. Rev. B43 (1991) 12074.

[13] M. E. Gershenson, P. M. Echternach, and H. M. Bozler, Phys. Rev. Lett. 74 (1995) 446.

[14] B. I. Belevtsev, E. Yu. Beliayev, V. V. Bobkov, and V. I. Glushko, Fiz. Niz. Temp. 21 (1995) 763 [Low Temp. Phys. 21 (1995) 592].

[15] B. I. Belevtsev, Yu. F. Komnik, and E. Yu. Beliayev, Fiz. Nizk. Temp. 21 (1995) 839 [Low Temp. Phys. 21 (1995) 646].

[16] A. V. Butenko, E. I. Bukhshtab, and V. V. Pilipenko, Fiz. Nizk. Temp. 10 (1984) 773 [Sov. J. Low Temp. Phys. 10 (1984) 407].

[17] M. Mostefa, D. Bourbie, and G. Olivier, Physica B160 (1989) 186.

[18] V. L. Nguen, B. Z. Spivak, and B. I. Shklovskii, Pis'ma v Zh. Eksp. Teor. Fiz. 41 (1985) 35 [JETP Letters 41 (1985) 42].

[19] B. I. Shklovskii and B. Z. Spivak, in Hopping Transport in Solids, eds. M. Pollak and B. I. Shklovskii (Elsevier Science Publishers B. V., New York, 1991).

[20] U. Sivan, O. Entin-Wohlman, and Y. Imry, Phys. Rev. Lett. 60 (1988) 1566.

[21] H. Bötger, V. V. Bryksin, F. Schulz, Phys. Rev. B49 (1994) 2447.

[22] M. Eto, Phys. Rev. B48 (1993) 4933.

[23] X. R. Wang and X. C. Xie, Europhys. Lett. 26 (1997) 1111. 


\section{FIGURE CAPTIONS}

Fig. 1. $\ln R$ versus $1 / T$ at $U=0.05 \mathrm{~V}(1), 0.10 \mathrm{~V}(2), 0.2 \mathrm{~V}(3), 0.5 \mathrm{~V}(4), 1.0 \mathrm{~V}$ (5).

Fig. 2. $\ln R$ versus $1 / U$ at $T=0.51 \mathrm{~K}(1), 2.0 \mathrm{~K}(2), 4.0 \mathrm{~K}(3), 7.0 \mathrm{~K}(4), 10.0 \mathrm{~K}(5)$.

Fig. 3. The relative variation of resistance in magnetic field at $T=5 \mathrm{~K}$. The curves correspond to $U=5 \mathrm{~V}(1), 2.5 \mathrm{~V}(2), 2.0 \mathrm{~V}(3), 1.5 \mathrm{~V}(4), 1.2 \mathrm{~V}(5), 1.0 \mathrm{~V}(6), 0.8 \mathrm{~V}(7), 0.5 \mathrm{~V}(8)$.

Fig. 4. The general picture of influence of $T$ and $U$ on film resistance. Bold lines correspond to the resistances $R_{\square}=1 \times 10^{7} \Omega(1), 1 \times 10^{6} \Omega(2), 1 \times 10^{5} \Omega(3), 1 \times 10^{4} \Omega(4)$. Thin lines (between bold ones) represent the intermediate values of resistance $2 \times 10^{n}$ and $5 \times 10^{n}$ where $n=4,5$, or 6 . Upper thin line corresponds to $R_{\square}=5 \times 10^{3} \Omega$, lower one - to $2 \times 10^{7} \Omega$.

Fig. 5. The $-\Delta R(H) / R$ as a function of $H^{2}$ at $T=3 \mathrm{~K}(1), 4 \mathrm{~K}(2), 5 \mathrm{~K}(3), 6 \mathrm{~K}(4), 7 \mathrm{~K}(5), 8 \mathrm{~K}$ (6), $10 \mathrm{~K}(7), 12 \mathrm{~K}(8)$. For each temperature these dependences correspond to the minimal value of applied voltage $U$. The lines correspond to the relationship $-\Delta R(H) / R \propto H^{2}$.

Fig. 6. The dependence $A=f(1 / T)$. The function $A(T)$ describes the temperature dependence of MR of investigated film in accordance with the expression $\Delta R / R=-A(T) H^{2}$. 
This figure "fig1.gif" is available in "gif" format from: http://arxiv.org/ps/cond-mat/9808323v1 
This figure "fig2.gif" is available in "gif" format from: http://arxiv.org/ps/cond-mat/9808323v1 
This figure "fig3.gif" is available in "gif" format from: http://arxiv.org/ps/cond-mat/9808323v1 
This figure "fig4.jpg" is available in "jpg" format from: http://arxiv.org/ps/cond-mat/9808323v1 
This figure "fig5.gif" is available in "gif" format from: http://arxiv.org/ps/cond-mat/9808323v1 
This figure "fig6.gif" is available in "gif" format from: http://arxiv.org/ps/cond-mat/9808323v1 